\title{
Relationship of tooth loss to mild cognitive impairment among middle-aged Mongolians: Mon-Timeline study
}

Urangoo Ganbaatar ${ }^{1}$, Oyuntuya Gantulga ${ }^{1}$, Puntsagdulam Byambajav ${ }^{2}$, Maralgua Och ${ }^{3}$, Ganjargal Ganburged ${ }^{4}$, Tsolmon Jadamba ${ }^{3,5}$, Byambasuren Dagvajantsan ${ }^{2 *}$, Oyuntugs Byambasukh ${ }^{1 *}$

${ }^{1}$ Graduate School, Mongolian National University of Medical Sciences, Ulaanbaatar, Mongolia

${ }^{2}$ Department of Neurology, School of Medicine, Mongolian National University of Medical Sciences, Ulaanbaatar, Mongolia

${ }^{3}$ Timeline Research Center, Ulaanbaatar, Mongolia

${ }^{4}$ Department of Orthodontics, School of Dental medicine, Mongolian National University of Medical Sciences, Ulaanbaatar, Mongolia

${ }^{5}$ Brain and mind institute, Mongolian Academy of Sciences, Ulaanbaatar, Mongolia

* Correspondence: oyuntugs@mnums.edu.mn; Tel.: +976-99017063

Received: 30 June 2021; Accepted: 18 October 2021; Published: 18 December 2021

Edited by: Battuvshin Lkhagvasuren (Mongolian National University of Medical Sciences, Mongolia)

Reviewed by: Buhari Ibrahim (Bauchi State University Gadau, Nigeria); Tuvshingerel Sandagdorj (National Cancer Center, Mongolia)

https://doi.org/10.31117/neuroscirn.v4i4.88

\begin{abstract}
Cognitive impairment is common in elderly people, so it is considered an ageing disorder. However, cognitive decline, including dementia, can also occur in middle-aged people. Cognitive impairment is associated with multiple risk factors. We hypothesised that tooth loss might also be a potential risk factor among Mongolians, as oral health problems are one of the significant health issues in Mongolia, especially in middle-aged people. In this cross-sectional study, we used the baseline data from the Mon-Timeline cohort study, including people older than 40 years of age $(n=279)$. The amount of tooth loss was assessed by a trained researcher. Mild cognitive impairment $(\mathrm{MCl})$ was defined as those participants scoring a total of $\leq 24$ points based on the Mini-Mental State Examination (MMSE). Unadjusted analysis showed that having more tooth loss $(>10)$ increased the risk of $\mathrm{MCl}$ by an odds ratio of 3.03 (1.49-6.17), as compared with having less tooth loss ( $\leq 10)$. Even after adjusting for covariates, the association remained significant, suggesting that tooth loss is associated with $\mathrm{MCl}$ risk, independent of age, education, and other socioeconomic factors. There was no significant interaction effect of age in the association between tooth loss and MMSE scores. In conclusion, tooth loss may play a role in developing cognitive decline, especially in the early onset of dementia. Further studies are needed to investigate whether early tooth loss at younger ages is associated with dementia, especially among middle-aged people.
\end{abstract}

Keywords: Tooth loss; mild cognitive impairment; MMSE; Mon-Timeline study;

C2021 by Ganbaatar et al. for use and distribution according to the Creative Commons Attribution (CC BY-NC 4.0) license (https://creativecommons.org/licenses/by-nc/4.0/), which permits unrestricted non-commercial use, distribution, and reproduction in any medium, provided the original author and source are credited.

\subsection{INTRODUCTION}

Cognitive impairment is one of the most common disorders in the general population that reduces the quality of life (Bárrios et al., 2013). Mild cognitive impairment $(\mathrm{MCl})$ is defined as an ageing-related cognitive decline, which can also progress to illnesses 
such as dementia (Mariani et al., 2007; Prince et al., $\underline{2013}$ ). It has been estimated that 50 million people have dementia, with an increase of nearly 10 million new cases every year (World Health Organization, 2019). This increase is mainly attributed to the rising number of those who have dementia in low- and middle-income countries, which are estimated to account for nearly $60 \%$ of all dementia cases (World Health Organization, 2019). In Mongolia, the prevalence of cognitive impairment, $39.7 \%$ of Mongolian adults over 60 years old had cognitive impairment in 2011 (Oyunkhand, 2011). The study used the same method to identify cognitive impairment (ICD 10, G31. 84), which is defined as cognitive impairment when Mini-Mental State Examination (MMSE) score 24 in our study (Folstein et al., 1975). Cognitive impairment is caused by multiple factors, including ageing, vascular diseases, and neurodegenerative diseases such as Alzheimer disease and other forms of dementia (Biessels et al., 2006; Sharp et al., 2011). Furthermore, many other risk factors for the development of cognitive impairment are reported in the literature (Beydoun et al., 2008; Fang et al., 2018; Munkhsukh et al., 2021; Namjil et al., 2021; Tonsekar et al., 2017). It is crucial to identify potential risk factors, especially for multiple countries and territories, to improve the prevention of dementia. Tooth loss is a risk factor for the development of cognitive impairment, especially in low- and middle-income countries (Fang et al., 2018; Tonsekar et al., 2017).

In Mongolia, oral health-related diseases are highly prevalent (Chinzorig et al., 2019; Jargaltsogt et al., 2018; Karvonen et al., 2003; Mongolian Government, 2006). The prevalence of dental caries, especially, is considerably higher in children. According to a study conducted in 2019, the prevalence of dental caries (ICD code K02.1-9) among children under 18 years of age is 89.3\% (Chinzorig et al., 2019), showing that the prevalence of this disease has not significantly changed from 93.2\% in 1993 (Jargaltsogt et al., 2018) and that the burden of dental caries has not changed in the last 20 years. Therefore, we hypothesised that tooth loss might be one of the potential risk factors for cognitive impairment among Mongolians, as oral health problems are one of the major health issues facing Mongolians. Although tooth loss may be associated with ageing, fewer teeth from a young age may be a risk factor for cognitive impairment. It is prevalent for people under the age of 40 to have 20-24 teeth (Tonsekar et al., 2017). However, Mongolians have already lost 6-7 teeth since 20 (Chinzorig et al., 2019; Jargaltsogt et al., 2018). This study explored the association between tooth loss and cognitive impairment within a representative data set of middle-aged people.

\subsection{MATERIALS AND METHODS}

\subsection{Data source and study population}

The Mon-Timeline is a multidisciplinary, prospective, population-based cohort study in Mongolia investigating various health problems among Mongolians, especially concerning oral and mental health. The sample size was calculated based on the prevalence of dental caries $(\mathrm{P}=89.3 \%)$ in the previous study (Chinzorig et al., 2019), assuming a 95\% confidence interval ( $\mathrm{Z}=1.96)$ with a $5 \%$ acceptable margin of error, which gave us a sample size of 1780 persons. A total of 1860 randomly selected Mongolians of rural and urban areas, including 18 clusters, participated in this study. In this cross-sectional study, we used the baseline data from the Mon-Timeline cohort, including people older than 40 years of age $(n=748)$. The exclusion criterion was any missing data related to the primary determinant and outcome $(n=124)$ : oral health and cognitive examination.

Furthermore, participants with a history of head trauma, stroke, heart failure or renal failure were excluded $(n=118)$. Another exclusion criterion was any psychiatric disorders such as depression diagnosed by a psychiatrist during the examination $(n=58)$. The psychiatrist also asked about previous mental illnesses and checked the patient reports. Additionally, we asked how participants would assess their health status (poor, fair, good or excellent); those with their health status assessed as poor were excluded $(n=157)$ based on a previously published method (Amaral et al., 2019) to rule out possible coexisting diseases. Ultimately, 279 participants were included in the current study.

The study was approved by the medical ethical committee of the Mongolian National University of Medical Sciences (METc 2020/3-05). According to the Helsinki declaration, all participants provided their written informed consent.

\subsection{Variables and measurements}

Independent and dependent variables: The amount of tooth loss was assessed by a trained researcher. Based on previous studies which categorised the tooth loss in relation to cognitive impairment (Saito et al., 2018; Tsai et al., 2020), the study participants were divided into two groups, according to the number of teeth lost: (1) Group 1 included participants with up to 10 teeth lost $(<10)$, and (2) Group 2 included participants with ten and more teeth lost $(\geqslant 10)$. Cognitive function was 
evaluated with the MMSE (Folstein et al., 1975). The MMSE consists of 11 items, including orientation (2), retention (1), attention and calculation (1), recall (1), language (5), and copying (1); with a total score of 30 points. $\mathrm{MCl}$ was defined as the participants scoring 24 points or above.

Covariates and other variables: The level of education was categorised as low, medium, and high. Participants were classified as married or cohabiting and single as a dichotomous variable. We made a group according to family income based on the distribution of the variable: low, medium, and high. We categorised all participants into groups of non-smokers and smokers according to smoking status. Daily fruit and vegetable intakes were evaluated using the Food Frequency Questionnaire (Ministry of Health Mongolia, 2013). Physical activity was assessed using the Global Physical Activity Questionnaire (Armstrong \& Bull, 2006). The level of physical activity and intake of fruits and vegetables were categorised as sufficient and insufficient, separately. 'Participants' body weight (in $\mathrm{kg}$ ) and height (in $\mathrm{cm}$ ) were measured by well-trained assistants using a standardised protocol, and Body Mass Index (BMI; $\mathrm{kg} / \mathrm{m}^{2}$ ) was subsequently calculated.

\subsection{Statistical analysis}

The study characteristics were expressed as means with a standard deviation (S.D.) for normally distributed variables tested by histogram and z-scores and as numbers with percentages in cases of categorical data.

' 'Student's T-test and Pearson Chi-Square test were used to compare grouping variables. Estimated MMSE was calculated according to tooth loss and age groups using age-adjusted Analysis of Variance (ANOVA). Furthermore, a two-way ANOVA was used to compare the difference between means of MMSE according to groups for tooth loss age. The study population was categorised according to age $(<60$ and $\geqslant 60$ years of age) because cognitive decline, especially ageing related $\mathrm{Cl}$, is common for people $\geqslant 60$ years of age (World Health Organization, 2019). Spearman's correlation coefficient was calculated to evaluate associations of the amount of tooth loss and age with MMSE scores.

Binary logistic regression analysis was performed to evaluate the association between tooth loss and $\mathrm{MCl}$. Odds ratio (OR) was reported with a 95\% confidence interval (Cl). Analysis was adjusted for age, which included age and square of age (age ${ }^{2}$, because age may be linearly related to independent and dependent variables), education, marital status and family income, diet and physical activity. The determinants consisted of two categories of tooth loss ( $<10$ and $\geqslant 10)$, with $<10$ as the reference group for regression analysis. Furthermore, the interaction effect was tested with age in the association of tooth loss with outcome variables. Additionally, given that age may reflect the cognitive decline of ageing, Spearman's partial correlation coefficient was performed for the individual MMSE components. All statistical analyses were performed using IBM SPSS V.22.0 (Chicago, IL) and GraphPad Prism V.4.03 (San Diego, CA). A two-sided statistical significance was set at $p<0.05$ for all tests.

\subsection{RESULTS}

The number of people with tooth loss $\geqslant 10$ accounted for $45.5 \%(n=127)$ of the total population. People with tooth loss $\geqslant 10$ were older (Table 1) and more likely to have lower MMSE scores (Table 2). According to gender, socioeconomic factors, lifestyle characteristics, and $\mathrm{BMI}$, there were no significant differences between people with and without tooth loss of $\geqslant 10$.

MMSE scores differed statistically depending on the amount of tooth loss and age. Although the lower MMSE scores observed in tooth loss $\geqslant 10$ groups within both age groups were highlighted, this was especially clear in the older age group (Table 2). These differences were significant when tested using a two-way ANOVA: mean MMSE was significantly lower in the tooth loss $\geqslant$ 10 and $\geqslant 60$-year group when compared with other groups. Furthermore, age-adjusted MMSE scores were significantly different throughout tooth loss groups: 27.5 and 24.3 for people with tooth loss $<10$ and $\geqslant 10$, respectively $(p<0.001)$.

' 'Spearman's correlation analysis yielded that the amount of tooth loss was inversely correlated with MMSE scores $(r=-0.191, p<0.0001)$. Furthermore, age was significantly correlated to MMSE scores $(r=-0.306$, $p<0.0001)$ but positively correlated to the number of teeth loss $(r=0.168, p<0.0001)$.

People with $\mathrm{MCl}$ (MMSE $\geqslant 24$ ) accounted for $20.1 \%$ $(n=56)$ of the total population. The higher presence of $\mathrm{MCl}$ was observed in tooth loss $\geqslant 10$ groups within both age groups, especially in the older age group (Figure 1 ). The prevalence of $\mathrm{MCl}$ was $9.2 \%(n=11)$ and $18.2 \%(n=6)$ in tooth loss $<10$ groups while it was $25.0 \%(n=23)$ and $45.2 \%(n=16)$ in tooth loss $\geqslant 10$ groups in people aged $<60$ and $\geqslant 60$ years of age respectively.

Logistic regression analysis showed that having more tooth loss $(\geqslant 10)$ increased the risk of $\mathrm{MCl}$ by an OR of 
Table 1: General characteristics of the study population.

\begin{tabular}{lccc}
\hline Characteristics & Tooth loss $(<\mathbf{1 0})(\mathrm{n}=152)$ & Tooth loss $(\geq \mathbf{1 0})(\mathrm{n}=127)$ & $\boldsymbol{P}$-value \\
\hline Age (year) & $53.8 \pm 9.7$ & $55.4 \pm 7.6$ & $\mathbf{0 . 0 0 4}$ \\
Age category & & & \\
$\quad<60(\mathrm{n}=211)$ & $119(78.3)$ & $92(72.4)$ & 0.150 \\
$\quad \geq 60(\mathrm{n}=68)$ & $33(21.7)$ & $35(27.6)$ & \\
Male gender, $\mathrm{n}(\%)$ & $46(23.8)$ & $25(29.1)$ & 0.217 \\
Education: low level, $\mathrm{n}(\%)$ & $46(23.8)$ & $25(29.1)$ & 0.556 \\
Married or cohabitant, $\mathrm{n}(\%)$ & $26(13.5)$ & $18(20.9)$ & 0.082 \\
Family income level: Low, $\mathrm{n}(\%)$ & $68(35.2)$ & $34(39.5)$ & 0.581 \\
Current smokers, $\mathrm{n}(\%)$ & $28(14.5 \%)$ & $19(22.1 \%)$ & 0.291 \\
Alcohol use, $\mathrm{n}(\%)$ & $48(24.9)$ & $29(33.7)$ & 0.084 \\
Fruit and vegetables use: Insufficient, $\mathrm{n}(\%)$ & $69(35.8)$ & $27(31.4)$ & 0.285 \\
Physically inactive, $\mathrm{n}(\%)$ & $29(15.1)$ & $13(15.3)$ & 0.550 \\
Body mass index $\left(\mathrm{kg} / \mathrm{m}^{2}\right)$ & $29.97 \pm 5.12$ & $29.31 \pm 5.51$ & 0.404 \\
\hline
\end{tabular}

Data are presented as mean \pm S.D. and number (percentages, \%).

Table 2: Association between tooth loss and mean of MMSE scores by age

\begin{tabular}{lcccc}
\hline Category & \multicolumn{3}{c}{ Mean \pm SD } & Age-adjusted mean \pm S.E. \\
\cline { 2 - 5 } & Total $(\mathbf{n}=\mathbf{2 7 9})$ & $<\mathbf{6 0}$ years $(\mathbf{n}=\mathbf{2 1 1})$ & $\mathbf{2 6 0}$ years $(\mathbf{n}=\mathbf{6 8})$ & Total \\
\hline Tooth loss $<10(\mathrm{n}=152)$ & $27.4 \pm 3.4$ & $28.0 \pm 3.0(\mathrm{n}=119)$ & $26.1 \pm 3.1(\mathrm{n}=92)$ & $27.5 \pm 0.3$ \\
Tooth loss $\geq 10(\mathrm{n}=127)$ & $25.7 \pm 4.2$ & $27.0 \pm 4.3(\mathrm{n}=33)$ & $22.5 \pm 4.8(\mathrm{n}=35)$ & $24.3 \pm 0.4$ \\
P-value* & $<0.0001$ & 0.002 & 0.001 & - \\
P-value** & - & & $<0.0001$ & $<0.0001$ \\
Age $x$ tooth loss interaction P-value $=0.045$ & & & \\
* Student-T-test, ${ }^{* *}$ Two-way ANOVA &
\end{tabular}

Table 3: Association of tooth loss and mild cognitive impairment.

\begin{tabular}{lcccc}
\hline \multirow{2}{*}{ Models } & \multicolumn{3}{c}{ Risk of Mild cognitive impairment } \\
\cline { 2 - 5 } & \multicolumn{3}{c}{$<\mathbf{6 0}$ years } & \multicolumn{2}{c}{$\geq \mathbf{6 0}$ years } \\
\cline { 2 - 5 } & $\mathrm{OR}(95 \% \mathrm{Cl})$ & $P$-value & $\mathrm{OR}(95 \% \mathrm{Cl})$ & $P$-value \\
\hline Unadjusted & $3.27(1.50-7.14)$ & $\mathbf{0 . 0 0 3}$ & $3.79(1.25-11.46)$ & $\mathbf{0 . 0 1 8}$ \\
Adjusted for age* & $2.81(1.25-6.30)$ & $\mathbf{0 . 0 1 2}$ & $4.86(1.39-16.90)$ & $\mathbf{0 . 0 1 3}$ \\
Adjusted for age*, gender and education & $2.82(1.23-6.44)$ & $\mathbf{0 . 0 1 4}$ & $5.34(1.40-20.34)$ & $\mathbf{0 . 0 1 4}$ \\
Adjusted for age*, gender and SES factors & $2.74(1.19-6.28)$ & $\mathbf{0 . 0 1 7}$ & $8.61(1.93-38.44)$ & $\mathbf{0 . 0 0 5}$ \\
\hline
\end{tabular}

Data are presented as OR with $\mathrm{Cl} .{ }^{*} \mathrm{Age}$ and square of age. SES, Socioeconomic status factors which includes education, marital status and family income. 
Table 4: Correlations of tooth loss and age with individual components of MMSE

\begin{tabular}{|c|c|c|c|}
\hline Total MMSE and domain scores & Tooth loss & Age & Tooth loss (age-adjusted) \\
\hline Orientation to time & $-0.180 * *$ & -0.038 & $-0.217^{* *}$ \\
\hline Orientation to place & -0.112 & -0.079 & -0.168 \\
\hline Retention & -0.115 & $-0.143^{*}$ & -0.123 \\
\hline Calculation and attention & $-0.169 *$ & $-0.223 * *$ & $-0.158 *$ \\
\hline Recall & -0.111 & $-0.308^{* * *}$ & -0.118 \\
\hline Language expression /naming/ & $-0.150^{*}$ & -0.120 & $-0.168 *$ \\
\hline Language expression /repetition/ & $-0.183 * *$ & $-0.150^{*}$ & $-0.170^{*}$ \\
\hline Language comprehension /verbal/ & $-0.162 *$ & $-0.131 *$ & $-0.139 *$ \\
\hline Language comprehension /written/ & $-0.146 *$ & -0.022 & $-0.187 * *$ \\
\hline Language expression /sentence writing / & $-0.182 * *$ & -0.086 & $-0.154^{*}$ \\
\hline Copying & $-0.213 * *$ & $-0.235 * * *$ & $-0.180 * *$ \\
\hline Total MMSE score & $-0.191 * *$ & $-0.306 * *$ & $-0.234^{* * *}$ \\
\hline
\end{tabular}

Data is presented as Spearman's correlation coefficients. ${ }^{*} p<0.05,{ }^{* *} p<0.01,{ }^{* * *} p<0.001$

\section{$<60$ years}
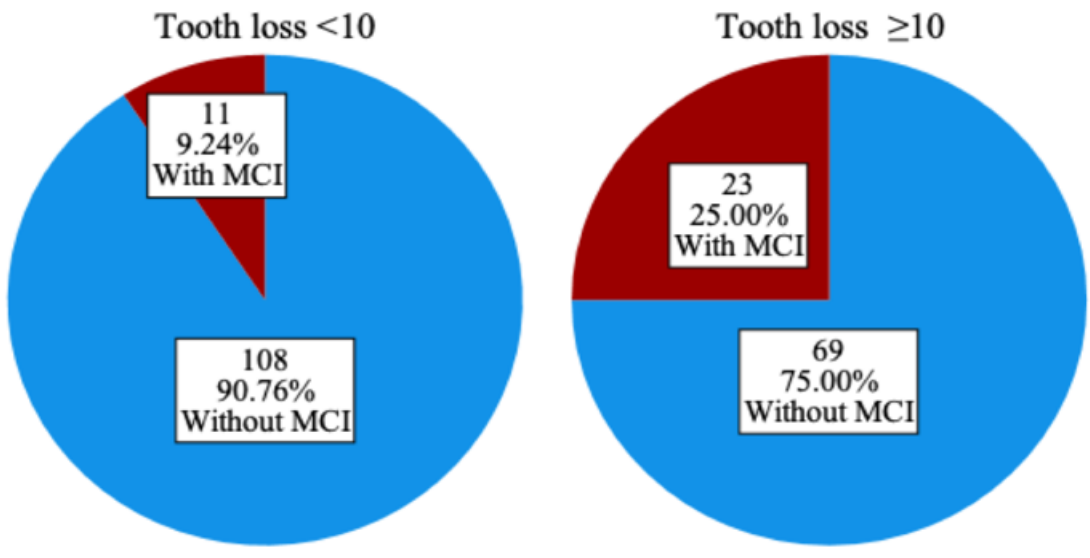

\section{$\geq 60$ years}

Tooth loss $<10$

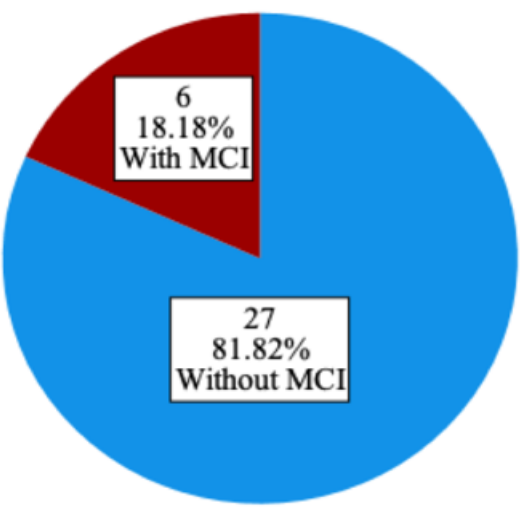

Tooth loss $\geq 10$

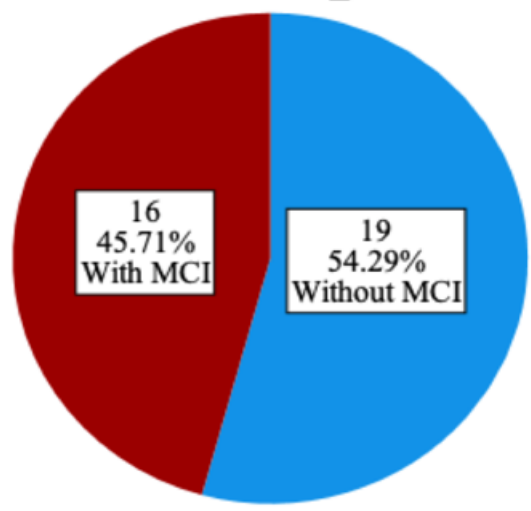

Figure 1: The presence of $\mathrm{MCl}$, according to age and tooth loss group. 
3.27 (1.50-7.14) and 3.79 (1.25-11.46) for people aged $<60$ years and $\geq 60$ years, respectively, as compared with having less tooth loss $(<10)$. This association remained significant after adjusting for age in both age groups (Table 3 ). However, the OR increased in the older age group while it decreased in the younger age group, suggesting that tooth loss, rather than age, may play a role in developing $\mathrm{MCl}$ in middle-aged people. Moreover, the association remained significant after further adjustments; thereby, suggesting that tooth loss is associated with $\mathrm{MCl}$ risk, independent of gender, education, marital status and family income, diet and physical activity.

In an additional analysis, we compared tooth loss and age with individual MMSE domain scores, separately, using Spearman's correlation to test the independent association of tooth loss with cognitive impairment. Furthermore, the correlation between tooth loss and MMSE (before adjusted for age $r=-0.191$ and $p<0.01$ ) remained significant and unchanged after adjusting for age $(r=-0.234$ and $p<0.001$, Table 4). The amount of tooth loss was more associated with orientation and language expression domains, while age was associated with retention and recall domains $(r=-0.111, p=0.213$ for tooth loss and $r=-0.308, p<0.001$ for age); thereby, suggesting that tooth loss might be associated with $\mathrm{MCl}$ via different pathways (Table 4).

\subsection{DISCUSSION}

This study found that multiple tooth loss is significantly associated with an increased risk of cognitive decline independent of age. Moreover, the association between tooth loss and the presence of $\mathrm{MCl}$ was found to be independent of gender and socioeconomic and lifestyle factors, such as education, marital status, family income, smoking, alcohol use, diet and physical activity.

In accordance with our results, previous studies have found an independent association between the number of teeth and cognitive function (Fang et al., 2018; Tonsekar et al., 2017). For instance, a Japanese 4-year follow-up study including 140 older adults who were evaluated with MMSE (MMSE $\geqslant 24$ ) found that cognitive impairment (MMSE $\leq 24)$ was more developed in participants with 0-9 teeth lost than those with $\geqslant 10$ teeth lost (Saito et al., 2018). A Taiwanese populationbased $(n=43026)$ retrospective study found that patients with more teeth extracted had a greater risk of dementia (Tsai et al., 2020). Both tooth loss and cognitive function are caused and influenced by multiple factors, such as socioeconomic factors and periodontal diseases (Ismail et al., 2019; Reyes-Ortiz et al., 2013; Tonsekar et al., 2017). Therefore, studies tested the association between tooth loss and cognition impairment with various confounding variables. In most of the studies, the association yielded significant results; but in a few studies, the association was mediated by age and socioeconomic factors (Ismail et al., 2019; Matthews et al., 2011). In our study, the association was significant after adjustment for various factors, such as education level, family income, marital status, smoking, alcohol use, diet and physical activity. The significant association between tooth loss and cognitive impairment has been proved by studies that explored various mechanisms in the pathogenesis of oral healthrelated cognitive impairment (Aida et al., 2006; Kornman et al., 1997; McGeer \& McGeer, 2001; Tonsekar et al., 2017). Studies concluded that the principal exploration involves masticatory dysfunction, which may occur due to tooth loss; thereby, causing decreases in the maximum bite force and occlusal contact area (Lexomboon et al., 2012; Tonsekar et al., 2017).

Indeed, cognitive decline is common in elderly people and is therefore largely considered an ageing disorder (Mariani et al., 2007; Prince et al., 2013; World Health Organization, 2019). However, cognitive decline, including dementia, can also occur in middle-aged people (World Health Organization, 2019). Especially in developing countries, the prevalence of early-onset dementia is increasing (World Health Organization, 2019). However, only limited studies have been conducted on middle-aged people. Our study included primarily middle-aged people and found a significant association between tooth loss and cognitive decline. In this study, the association was stronger in the older age group ( $\geq 60$ years), potentially explainable by the fact that cognitive decline is common in elderly people. Tooth loss also occurs more often in elderly people. However, there was no significant association between age and tooth loss in our study, as even middle-aged people were found to have a smaller number of teeth. In a stratified analysis, the association of tooth loss and $\mathrm{MCl}$ was significant in middle-aged people even after adjusting for age. Furthermore, when we tested the association of tooth loss with individual domain scores of MMSE compared to age. Tooth loss and age were differently associated with different domains of MMSE indicating that the development of cognitive decline may be different from age-induced cognitive decline due to tooth loss. Finally, we suggest that tooth loss may play a role in developing cognitive decline, especially for early-onset dementia. Further studies are needed to 
investigate whether early tooth loss at younger ages is associated with dementia, especially in middle-aged people.

The above-mentioned tooth loss is a risk factor for the development of cognitive impairment, thereby suggesting the importance of the presence of teeth in cognitive abilities. This is the first study to explore the association of tooth loss with the presence of cognitive impairment in Mongolia, showing that having a smaller number of teeth may be a considerable risk factor for cognitive impairment. Unfortunately, a recent study showed that no significant improvements in dental care had been observed during the last 20 years (Chinzorig et al., 2019; Mongolian Government, 2006). Dental care service was firstly studied in Mongolia in 1993, which showed a high prevalence of dental caries (93.2\%) (Jargaltsogt et al., 2018). The latest study showed that the prevalence of dental caries was $89.3 \%$ in 2019 (Chinzorig et al., 2019). Furthermore, this and other studies have shown that the risk of dental caries is even higher among Mongolian children (Chinzorig et al., 2019; Jargaltsogt et al., 2018; Karvonen et al., 2003; Mongolian Government, 2006). More than half of the children under 18 were found to have visited dental clinics because of toothache (Chinzorig et al., 2019). One of the causes for this poor oral health is urbanisation, whereby a traditional milk-based diet was changed to a milk-based deficient diet (Karvonen et al., 2003). This suggests that there might be a difference between urban and rural areas in the prevalence of dental caries. However, a recent study found no differences in dental caries prevalence among urban and rural areas (Chinzorig et al., 2019; Mongolian Government, 2006). While the high prevalence of caries was explained by poor knowledge of parents regarding oral hygiene care for their children, this has not been confirmed by recent studies. The population's brushing habits remain poor regardless of parental education and socioeconomic status (Chinzorig et al., 2019). Therefore, practical approaches to improve dental and oral hygiene need to be implemented nationwide to reduce the persistent high caries occurrence in Mongolia.

The main strength of our study is its use of a representative data set of middle-aged people.
Nevertheless, there are some limitations to our research. Our study design is cross-sectional. It is, therefore, not possible to rule out reverse causality. In addition, cognitive decline is typical in elderly people; therefore, ageing-related tooth loss can be more prevalent in elderly people (Bárrios et al., 2013; Mariani et al., 2007; Prince et al., 2013). Although we adjusted for age, there still could be a possibility of residual confounding by age-related factors. Some studies found that ageing-related cognitive decline could cause poor oral health, resulting in fewer teeth (Henriksen et al., 2005). This indicates a possibility of reverse causality in the association between tooth loss and cognitive decline due to ageing. Accordingly, we aimed to explore the association between tooth loss and cognitive impairment within middle-aged people. Another limitation is that we could not fully control periodontal diseases, oral hygiene and causes of tooth loss, its duration and places. Finally, the observational nature of the study precludes us from concluding causality.

\subsection{CONCLUSIONS}

Multiple tooth loss is significantly associated with an increased risk of cognitive decline. Further studies are needed to investigate whether early tooth loss at younger ages is associated with dementia, especially in middle-aged Mongolian people. Furthermore, this study results suggest that it is crucial to improve the care for oral health for Mongolians.

Acknowledgements: The authors acknowledge and thank the Timeline cohort study team and the Mongolian National University of Medical Sciences for their support and all the study participants. We also thank the reviewers for their excellent critique of the manuscript and for helping us better our work.

Author Contributions: Ts.J, O.B., G.G. and B.D. conceived and designed the study; U.G., O.G., G.G. and P.B. collected data; U.G., O.G., O.B. and P.B. analysed the data; B.D., O.B., MO and U.G. wrote the paper; Ts.J, MO, B.D. and O.B. reviewed and edited the paper.

Conflicts of Interest: The authors declare no conflict of interest.

\section{References}

Aida, J., Ando, Y., Akhter, R., Aoyama, H., Masui, M., \& Morita, M. (2006). Reasons for permanent tooth extractions in Japan. Journal of Epidemiology, 16(5), 214-219. https://doi.org/10.2188/JEA.16.214

Amaral, C. A., Amaral, T. L. M., Monteiro, G. T. R., Vasconcellos, M. T. L., \& Portela, M. C. (2019). Hand grip strength: Reference values for adults and elderly people of Rio Branco, Acre, Brazil. PloS One, 14(1).

https://doi.org/10.1371/JOURNAL.PONE.0211452 
Armstrong, T., \& Bull, F. (2006). Development of the World Health Organization Global Physical Activity Questionnaire (GPAQ). Journal of Public Health 2006 14:2, 14(2), 66-70. https://doi.org/10.1007/S10389-006-0024-X

Bárrios, H., Narciso, S., Guerreiro, M., Maroco, J., Logsdon, R., \& de Mendonça, A. (2013). Quality of life in patients with mild cognitive impairment. Aging \& Mental Health, 17(3), 287-292. https://doi.org/10.1080/13607863.2012.747083

Beydoun, M. A., Beydoun, H. A., \& Wang, Y. (2008). Obesity and central obesity as risk factors for incident dementia and its subtypes: a systematic review and meta-analysis. Obesity Reviews : An Official Journal of the International Association for the Study of Obesity, 9(3), 204-218. https://doi.org/10.1111/J.1467-789X.2008.00473.X

Biessels, G., Staekenborg, S., Brunner, E., Brayne, C., \& Scheltens, P. (2006). Risk of dementia in diabetes mellitus: a systematic review. The Lancet. Neurology, 5(1), 64-74. https://doi.org/10.1016/S1474-4422(05)70284-2

Chinzorig, T., Aida, J., Cooray, U., Nyamdorj, T., Mashbaljir, S., Osaka, K., \& Garidkhuu, A. (2019). Inequalities in caries experience among mongolian children. International Journal of Environmental Research and Public Health, 16(20), 3892. https://doi.org/10.3390/ijerph16203892

Fang, W. L., Jiang, M. J., Gu, B. B., Wei, Y. M., Fan, S. N., Liao, W., Zheng, Y. Q., Liao, S. W., Xiong, Y., Li, Y., Xiao, S. H., \& Liu, J. (2018). Tooth loss as a risk factor for dementia: systematic review and meta-analysis of 21 observational studies. $B M C$ Psychiatry, 18(1). https://doi.org/10.1186/S12888-018-1927-0

Folstein, M. F., Folstein, S. E., \& McHugh, P. R. (1975). "Mini-mental state”. A practical method for grading the cognitive state of patients for the clinician. Journal of Psychiatric Research, 12(3), 189-198. https://doi.org/10.1016/00223956(75)90026-6

Henriksen, B. M., Engedal, K., \& Axéll, T. (2005). Cognitive impairment is associated with poor oral health in individuals in long-term care. Oral Health \& Preventive Dentistry, 3(4), 203-207. https://doi.org/10.3290/i.ohpd.a10824

Ismail, F., Muneer, M. U., Ahmed, A. R., Hussain, M. W., Khan, M. F., Quddos, M. U., \& Kashif, M. (2019). Factors affecting cognition in individuals with tooth loss. Annals of Dental Specialty, 7(4), 7-13.

Jargaltsogt, D., Bazar, O., Sharkhuu, M.-O., Altansukh, S., \& Bazar, A. (2018). The results of National Oral Health Survey, Mongolia. Central Asian Journal of Medical Sciences, 4(1), 61-68.

Karvonen, H. M., Nuutinen, O., Uusitalob, U., Sorvari, R., \& Ihanainen, M. (2003). Child nutrition and oral health in Ulaanbaatar. Nutrition Research, 23(9), 1165-1176. https://doi.org/10.1016/S0271-5317(03)00088-5

Kornman, K., Crane, A., Wang, H., di Giovine, F., Newman, M., Pirk, F., Wilson, T., Higginbottom, F., \& Duff, G. (1997). The interleukin-1 genotype as a severity factor in adult periodontal disease. Journal of Clinical Periodontology, 24(1), 72-77. https://doi.org/10.1111/J.1600-051X.1997.TB01187.X

Lexomboon, D., Trulsson, M., Wãrdh, I., \& Parker, M. G. (2012). Chewing ability and tooth loss: association with cognitive impairment in an elderly population study. Journal of the American Geriatrics Society, 60(10), 1951-1956. https://doi.org/10.1111/J.1532-5415.2012.04154.X

Mariani, E., Monastero, R., \& Mecocci, P. (2007). Mild Cognitive Impairment: A Systematic Review. Journal of Alzheimer's Disease, 12(1), 23-35. https://doi.org/10.3233/JAD-2007-12104

Matthews, J. C., You, Z., Wadley, V. G., Cushman, M., \& Howard, G. (2011). The association between self-reported tooth loss and cognitive function in the REasons for Geographic And Racial Differences in Stroke study: an assessment of potential pathways. Journal of the American Dental Association (1939), 142(4), 379-390. https://doi.org/10.14219/JADA.ARCHIVE.2011.0192

McGeer, P. L., \& McGeer, E. G. (2001). Polymorphisms in inflammatory genes and the risk of Alzheimer disease. Archives of Neurology, 58(11), 1790-1792. https://doi.org/10.1001/ARCHNEUR.58.11.1790

Ministry of Health Mongolia. (2013). Mongolian STEPS Survey on the Prevalence of Noncommunicable Disease and Injury Risk Factors 2013.

Mongolian Government. (2006). Mongolia National Oral Health Program.

Munkhsukh, M. U., Namjil, D., Byambajav, P., Angarag, E. A., Junai, S., Dagvajantsan, B., \& Byambasukh, O. (2021). Dementia risk among Mongolian population with type 2 diabetes: a matched case-control study. Journal of Integrative Neuroscience, 20(3), 659-666. https://doi.org/10.31083/J.JIN2003070

Namjil, D., Dagvajantsan, B., \& Byambasukh, O. (2021). Vascular cognitive impairment risk among Mongolian adults: An overview. Neuroscience Research Notes, 4(3Suppl), 17-20. https://doi.org/10.31117/NEUROSCIRN.V4I3SUPPL.99

Oyunkhand, R. (2011). A study on Mongolian elderly people. Mongolian National University of Medical Sciences.

Prince, M., Bryce, R., Albanese, E., Wimo, A., Ribeiro, W., \& Ferri, C. P. (2013). The global prevalence of dementia: a systematic review and metaanalysis. Alzheimer's \& Dementia : The Journal of the Alzheimer's Association, 9(1), 63-75.e2. https://doi.org/10.1016/J.JALZ.2012.11.007

Reyes-Ortiz, C. A., Luque, J. S., Eriksson, C. K., \& Soto, L. (2013). Self-reported tooth loss and cognitive function: Data from the Hispanic Established Populations for Epidemiologic Studies of the Elderly (Hispanic EPESE). Colombia Médica : CM, 44(3), 139. https://doi.org/10.25100/cm.v44i3.1248

Saito, S., Ohi, T., Murakami, T., Komiyama, T., Miyoshi, Y., Endo, K., Satoh, M., Asayama, K., Inoue, R., Kikuya, M., Metoki, H., Imai, Y., Ohkubo, T., \& Hattori, Y. (2018). Association between tooth loss and cognitive impairment in communitydwelling older Japanese adults: a 4-year prospective cohort study from the Ohasama study. BMC Oral Health, 18(1). 
https://doi.org/10.1186/S12903-018-0602-7

Sharp, S. I., Aarsland, D., Day, S., Sønnesyn, H., \& Ballard, C. (2011). Hypertension is a potential risk factor for vascular dementia: systematic review. International Journal of Geriatric Psychiatry, 26(7), 661-669. https://doi.org/10.1002/GPS.2572

Tonsekar, P. P., Jiang, S. S., \& Yue, G. (2017). Periodontal disease, tooth loss and dementia: Is there a link? A systematic review. Gerodontology, 34(2), 151-163. https://doi.org/10.1111/ger.12261

Tsai, Y. C., Wang, H. J., Wang, L. Y., Shaw, C. K., Lee, Y. P., Lin, M. C., \& Huang, C. H. (2020). Retrospective analysis of the association between tooth loss and dementia: a population-based matched case-control study. Community Dental Health, 37(1), 59-64. https://doi.org/10.1922/CDH 4609TSAl06

World Health Organization. (2019). Risk Reduction of Cognitive Decline and Dementia. In Risk Reduction of Cognitive Decline and Dementia: WHO Guidelines. World Health Organization. https://www.ncbi.nlm.nih.gov/books/NBK542796/ 